CLICdp-Conf-2017-013

26 June 2017

\title{
A Vertex and Tracking Detector System for CLIC
}

\author{
A. Nürnberg ${ }^{1) *}$ \\ On behalf of the CLICdp collaboration \\ ${ }^{*}$ CERN, Switzerland
}

\begin{abstract}
The physics aims at the proposed future CLIC high-energy linear $\mathrm{e}^{+} \mathrm{e}^{-}$collider pose challenging demands on the performance of the detector system. In particular the vertex and tracking detectors have to combine precision measurements with robustness against the expected high rates of beam-induced backgrounds. The requirements include ultra-low mass, facilitated by power pulsing and air cooling in the vertex-detector region, small cell sizes and precision hit timing at the few-ns level. A detector concept meeting these requirements has been developed and an integrated $R \& D$ program addressing the challenges is progressing in the areas of ultra-thin sensors and readout ASICs, interconnect technology, mechanical integration and cooling.
\end{abstract}

Talk presented at International Conference on Technology and Instrumentation in Particle Physics 2017 (TIPP2017), Beijing, China, 22-26 May 2017

(C) 2017 CERN for the benefit of the CLICdp Collaboration.

Reproduction of this article or parts of it is allowed as specified in the CC-BY-4.0 license.

1 andreas.nurnberg@cern.ch 


\title{
A Vertex and Tracking Detector System for CLIC
}

\author{
A. Nürnberg on behalf of the CLICdp collaboration \\ CERN, Geneva, Switzerland, andreas.nurnberg@cern.ch
}

\begin{abstract}
The physics aims at the proposed future CLIC high-energy linear $\mathrm{e}^{+} \mathrm{e}^{-}$collider pose challenging demands on the performance of the detector system. In particular the vertex and tracking detectors have to combine precision measurements with robustness against the expected high rates of beam-induced backgrounds. The requirements include ultra-low mass, facilitated by power pulsing and air cooling in the vertex-detector region, small cell sizes and precision hit timing at the few-ns level. A detector concept meeting these requirements has been developed and an integrated R\&D program addressing the challenges is progressing in the areas of ultra-thin sensors and readout ASICs, interconnect technology, mechanical integration and cooling.
\end{abstract}

\section{Introduction}

CLIC is a proposed future high-energy linear $\mathrm{e}^{+} \mathrm{e}^{-}$collider [1]. The high-precision physics aims pose challenging demands on the performance of the detector systems, including the vertex and tracking detector [2]. In particular, a precise determination of displaced vertices for efficient flavor tagging requires an impact parameter resolution of $\sigma\left(d_{0}\right)=5 \oplus 15 /\left(p[\mathrm{GeV}] \sin ^{\frac{3}{2}} \theta\right) \mu \mathrm{m}$ for the vertex detector, whereas the main requirement for the tracker is a transverse momentum resolution of $\sigma_{p_{\mathrm{T}}} / p_{T}^{2}=2 \times 10^{-5} \mathrm{GeV}^{-1}$ for high- $p_{\mathrm{T}}$ tracks above $100 \mathrm{GeV}$ in the central detector. At the same time, the material budget and power consumption have to be kept at a minimum. Further, background particles from beam-beam interactions can reach the detector and thus small cell sizes and precise hit timing in the vertex and tracking detector are necessary.

\section{Vertex and Tracking Detector Concepts}

To fulfill the requirements outlined in Section 1, silicon vertex and tracking detectors are foreseen for CLIC [3]. Both sub-detectors are illustrated in Fig. 1.

The vertex detector consists of three double layers in the barrel region, ranging from $31 \mathrm{~mm}$ to $70 \mathrm{~mm}$ in radius, and discs on each side of the detector. The material budget per detection layer of only $0.2 \% \mathrm{X}_{0}$ does not allow for liquid cooling of the detector. To extract the dissipated heat, forced air-flow is foreseen. The discs are arranged in a spiral geometry, to allow for better air-flow. To meet the required impact parameter resolution and flavor tagging efficiency, 
$3 \mu \mathrm{m}$ single point resolution has to be achieved throughout the vertex detector. Currently, planar and capacitively coupled hybrid pixel detectors are under consideration, e.g. $[4,5]$.

The main silicon tracker consists of 6 barrel layers, 7 inner discs and 4 outer flat discs, with material content per detection layer corresponding to $1-2 \% \mathrm{X}_{0}$ [3]. The tracking detector is divided into an inner and outer part by the support cylinder for the vacuum tube. The tracker radius is $1450 \mathrm{~mm}$. In total, the active area covered by the tracker is in the order of $100 \mathrm{~m}^{2}$. For this large area, monolithic solutions are considered, e.g. [6].

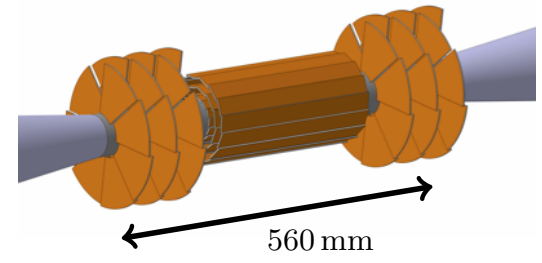

(a) Vertex detector

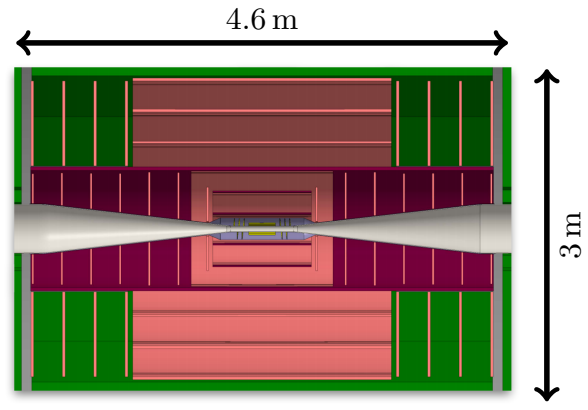

(b) Tracking detector

Fig. 1: Rendering of the vertex and tracking detectors as implemented in the CLICdet detector model [3].

The low duty cycle of the CLIC machine (312 bunches in $156 \mathrm{~ns}$ long bunch trains every $20 \mathrm{~ms}$ ) allows for pulsed power operation of the vertex and tracking detectors. This helps in reducing the average power consumption and enables the vertex detector to be air-cooled. For the large tracker volume, air-cooling can not easily be implemented, therefore liquid cooling is currently foreseen.

\section{Detector Performance}

Extensive simulation and engineering studies have been performed in order to optimize the detector layout and to demonstrate the technical feasibility of the proposed solutions.

\subsection{Flavor tagging}

The main goal of the vertex detector is the tagging of heavy quarks by the reconstruction of displaced decay vertices. Beauty- and charm-tagging performance has been chosen as benchmark for the detector design. Full simulation studies based on Geant4 $[7,8]$ as well as multivariate analyses using the LCFIPlus 
package [9] have been performed on various implementations of the detector. The variations have been guided by engineering constraints. Results from this detector optimization are illustrated in Fig. 2, while a more complete description can be found in $[10,11]$.

The impact of the variation of the material content per detector layer from $0.1 \% \mathrm{X}_{0}$ to $0.2 \% \mathrm{X}_{0}$ on the misidentification of $\mathrm{b}$ and light flavor backgrounds as function of the c-tagging efficiency is shown in Fig. 2a, and reveals an increase of the fake rate by $5 \%$ to $35 \%$. This demonstrates the importance of limiting the material budget of the detector. $0.2 \% \mathrm{X}_{0}$ per layer is considered to be realistically achievable taking technological and engineering constraints into account.

The relative performance of the spiral geometry in comparison to flat discs is illustrated in Fig. 2b, and shows only slightly reduced performance in some regions with lower coverage.

The slight benefit of 3 double layers in the barrel compared to 5 single layers as depicted in Fig. 2c can be explained by the small reduction on the material per layer due to shared support structures in the case of the double layer arrangement and the additional track measurement point.

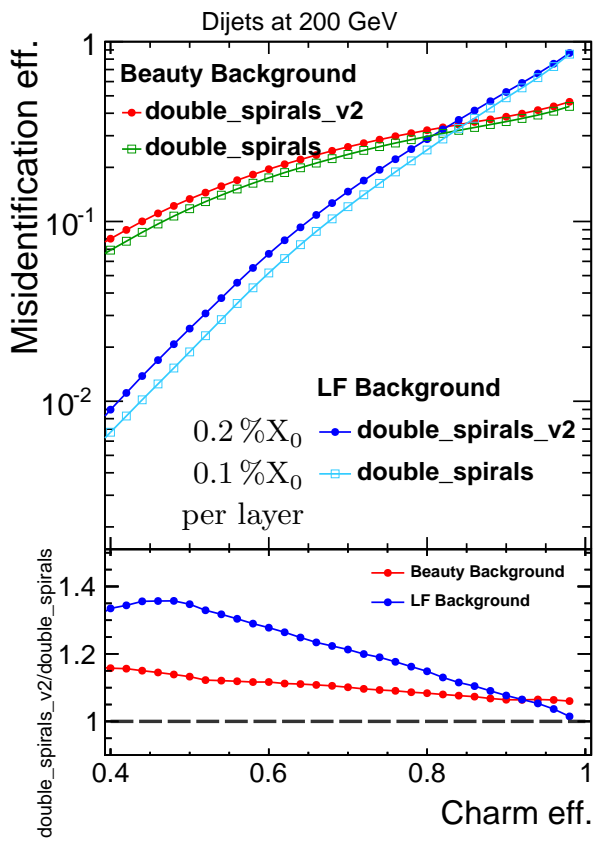

(a) Material budget

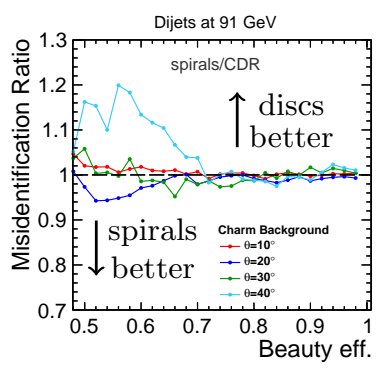

(b) Discs vs. spirals

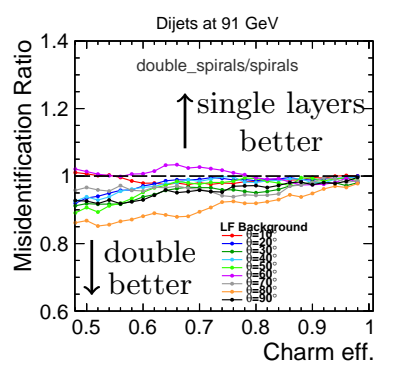

(c) Single vs. double layers

Fig. 2: Flavor tagging performance [10]. 


\subsection{Transverse momentum resolution}

The track momentum measurement is based on the measurement of the curvature of charged particle trajectories in a magnetic field. The achievable resolution is an interplay between the strength of the magnetic field, the tracker radius and the single point resolution of the detection layers. Optimization studies discussed in [3] lead to the choice of $\mathrm{B}=4 \mathrm{~T}, \mathrm{R}=1.5 \mathrm{~m}$ and a single point resolution $\sigma_{r \varphi}=7 \mu \mathrm{m}$ in the $r \varphi$-plane. Both, fast [12] and full simulation studies presented in Fig. 3 confirm that the transverse momentum resolution goal of $\sigma_{p_{\mathrm{T}}} / p_{T}^{2}=2 \times 10^{-5} \mathrm{GeV}^{-1}$ can be reached for high momentum tracks in the central detector.

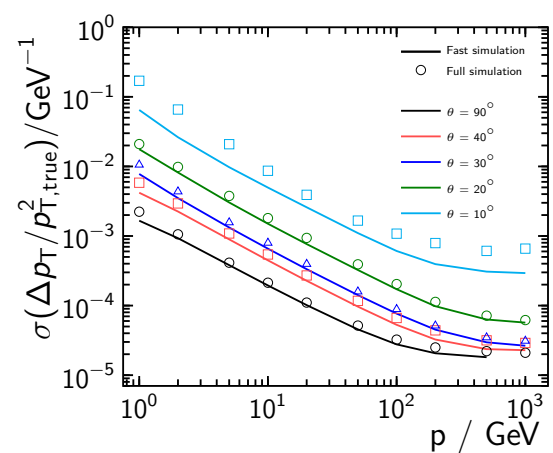

(a) Comparison between fast and full simulation results.

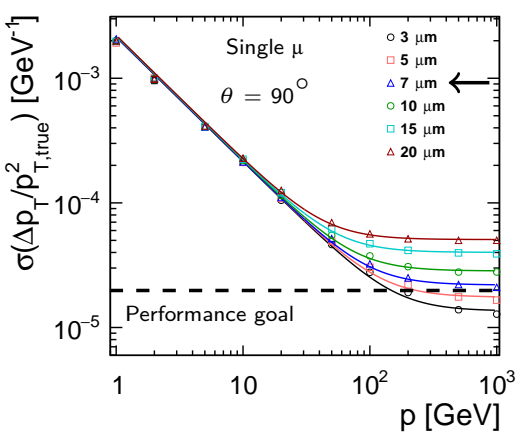

(b) Dependence on single point resolution [13].

Fig. 3: Transverse momentum resolution for single muons.

\subsection{Background occupancy}

To achieve a high luminosity of the CLIC machine, a strong focusing of the beams to very small beam sizes is needed. This leads to beam-beam interactions which result in background particles being created $[2,14]$. Particles from incoherent $\mathrm{e}^{+} \mathrm{e}^{-}$pair production as well as from $\gamma \gamma \rightarrow$ hadron events can reach the detector acceptance and create background hits in the tracking detectors. The cell sizes have to been chosen such, that the occupancy of the detectors is limited to $3 \%$ integrated over the full bunch train. This value is assumed to be tolerated by the track reconstruction algorithms. For the vertex detector, $25 \times 25 \mathrm{\mu m}^{2}$ pixels are assumed, and short strips/long pixels in the range of $1-10 \mathrm{~mm} \times 30-50 \mu \mathrm{m}$ are envisaged for the tracker [3]. Fig. 4 summarizes the occupancy of the vertex barrel layers and tracking detector discs due to beam-induced background particles obtained in a Geant4 based full detector simulation. It demonstrates that the 
occupancy can be limited to $3 \%$. Safety factors for the uncertainties related to the production and simulation uncertainties of the individual background processes have been applied [14, 13].

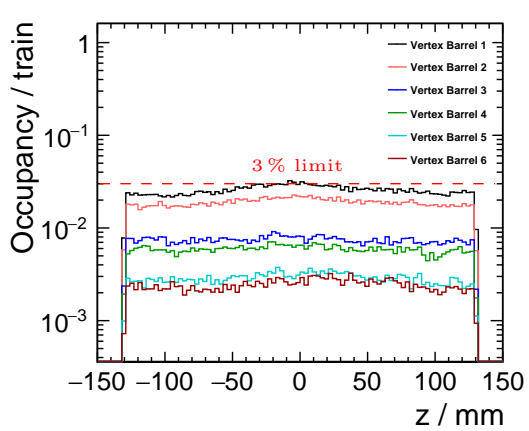

(a) Vertex barrel

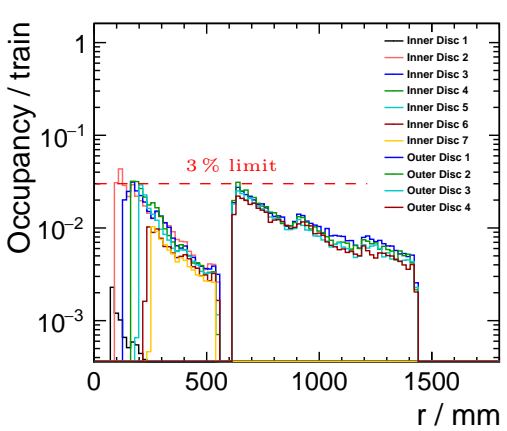

(b) Tracker discs

Fig. 4: Bunch train occupancies in the vertex and tracking detectors due to beam induced background hits from incoherent pair production and $\gamma \gamma \rightarrow$ hadron background processes [13], assuming $25 \times 25 \mathrm{\mu m}^{2}$ pixel size for the vertex detector and $1-10 \mathrm{~mm} \times 50 \mu \mathrm{m}$ for the tracker.

\section{Summary}

The design of the vertex and tracking detector for CLIC is driven by the stringent requirements on measurement precision, the limited material and power budget and the challenging background conditions. Simulation and engineering studies have demonstrated that a light-weight air-cooled vertex detector gives excellent flavor tagging performance, and that a large silicon tracker provides excellent track momentum measurement. Both are essential ingredients for the physics goals at CLIC. An integrated R\&D program addressing the technological challenges is progressing in the areas of ultra-thin sensors and readout ASICs, interconnect technology, mechanical integration and cooling, to show the feasibility of the proposed vertex and tracker detector concept.

\section{References}

[1] Aicheler, M., et al.: A Multi-TeV Linear Collider Based on CLIC Technology: CLIC Conceptual Design Report (2012), https://cds.cern.ch/record/1500095, CERN-2012-007

[2] CLIC Conceptual Design Report: Physics and Detectors at CLIC (2012), CERN2012-003 
[3] Alipour Tehrani, N., et al.: CLICdet: The post-CDR CLIC detector model (2017), http://cds.cern.ch/record/2254048, CLICdp-Note-2017-001

[4] Alipour Tehrani, N.: Test-beam measurements and simulation studies of thinpixel sensors for the CLIC vertex detector. Ph.D. thesis, ETH Zurich (2017), https://www.research-collection.ethz.ch/handle/20.500.11850/164813, Diss. ETH No. 24216

[5] Buckland, M.: Analysis and simulation of HV-CMOS assemblies for the CLIC vertex detector (2017), These proceedings.

[6] Munker, M.: Integrated CMOS sensor technologies for the CLIC tracker (2017), These proceedings.

[7] Agostinelli, S., et al.: Geant4 - a simulation toolkit. Nuclear Instruments and Methods in Physics Research Section A: Accelerators, Spectrometers, Detectors and Associated Equipment 506(3), 250 - 303 (2003)

[8] Allison, J., et al.: Geant4 developments and applications. IEEE Transactions on Nuclear Science 53(1), 270-278 (2006)

[9] Suehara, T., Tanabe, T.: LCFIPlus: A framework for jet analysis in linear collider studies. Nuclear Instruments and Methods in Physics Research Section A: Accelerators, Spectrometers, Detectors and Associated Equipment 808, 109 - 116 (2016), http://www.sciencedirect.com/science/article/pii/S0168900215014199

[10] Alipour Tehrani, N., Roloff, P.: Optimisation Studies for the CLIC VertexDetector Geometry (2014), https://cds.cern.ch/record/1742993, CLICdp-Note2014-002

[11] Alipour Tehrani, N.: Optimisation studies for the CLIC vertex-detector geometry. Journal of Instrumentation 10(07), C07001 (2015), http://stacks.iop.org/1748$0221 / 10 / \mathrm{i}=07 / \mathrm{a}=\mathrm{C} 07001$

[12] Regler, M., Valentan, M., Fruhwirth, R.: LiC Detector Toy 2.0 (Vienna Fast Simulation Tool for Charged Tracks), Users Guide http://www.hephy.at/project/ilc/lictoy/, HEPHY-PUB-863/08

[13] Nurnberg, A., Dannheim, D.: Requirements for the CLIC tracker readout (2017), https://cds.cern.ch/record/2261066, CLICdp-Note-2017-002

[14] Dannheim, D., Sailer, A.: Beam-Induced Backgrounds in the CLIC Detectors (2012), http://cds.cern.ch/record/1443516, LCD-Note-2011-021 